\title{
Phosphorus analogues of rubber
}

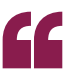

Who knows

what

phosphorus

might bring to

the table...but

it is sure to be

different
The prevalence of rubber - from both natural and synthetic sources - is evident in our everyday lives from car tyres to protective gloves. Now, writing in Angewandte Chemie, Derek Gates and co-workers report phosphorus versions of rubber made by polymerizing monomers containing both a phosphorus-carbon and a carbon-carbon double bond. This replacement of carbon with phosphorus, and the subsequent synthesis and detailed characterization of two polymers, poly(1-phospha-1,3-butadiene) and poly(1-phosphaisoprene), comes more than a

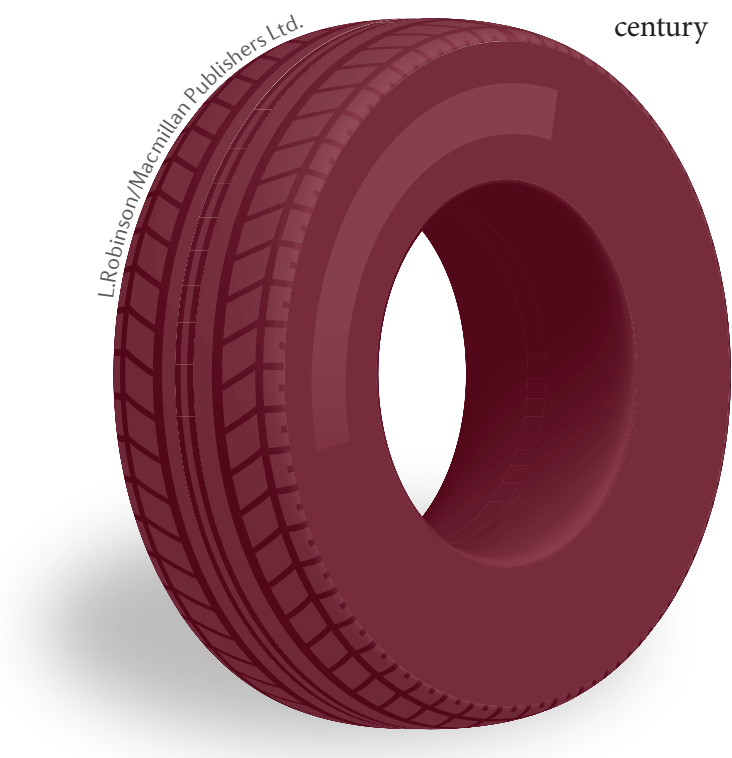

after the development of polymerization routes for the all-carbon equivalent monomers.

Even though the chemical structures of natural/synthetic rubbers and the phosphorus analogues are similar, the physical properties and chemical function of the materials are different. For example, the glass transition temperatures of the phosphorus-containing polymers are much higher than for rubber. "These new materials are likely to display rubbery properties only at high temperatures, or alternatively, they may not display rubbery properties at all," says Gates.

Poly(1-phosphaisoprene), which is a yellow glass at room temperature, can be made either in a one-pot method or in a two-step process in which the 1-phosphaisoprene monomer is isolable. Both methods involve a Grignard reagent, and following purification, they produce polymers of modest to high molecular weight. The anion-initiated polymerization largely occurs through the carboncarbon double bond, and only a very small fraction of the phosphorus is detected in the backbone of the polymers. The phosphorus-carbon double bond remains in the side chains of the polymer and imparts chemical functionality to the material. In contrast to the traditional carbon-containing polymers that are largely used for passive applications, such as coatings and plastics, the presence of phosphorus in the side chains opens up the possibility of making active materials that can undergo further reactions or respond to chemical or environmental stimuli.

"We believe that phosphoruscontaining co-polymers that may be produced in the future will be very interesting," explains Gates. "For example, if we were to replace isoprene with 1-phosphaisoprene in the production of butyl rubber, the resultant polymer would not only have crosslinking points in the form of the double bonds, but also chemically functionalizable phosphorus atoms." The phosphorus atoms could lead to materials with antioxidant behaviour, or for use as flame retardants or to bind metals. "Who knows what phosphorus might bring to the table... but it is sure to be different," concludes Gates.

Alison Stoddart

ORIGINAL ARTICLE Dück, K., Rawe, B. W.

Scott, M. R. \& Gates, D. P. Polymerization of

1-phosphaisoprene: synthesis and characterization of a chemically functional phosphorus version

of natural rubber. Angew. Chem. Int. Ed.

http://dx.doi.org/10.1002/anie.201703590 (2017) 